Available online on 15.05.2018 at http://jddtonline.info
Journal of Drug Delivery and Therapeutics
Open Access to Pharmaceutical and Medical Research
$\begin{gathered}\text { (0) 2011-18, publisher and licensee JDDT, This is an Open Access article which permits unrestricted non- } \\ \text { commercial use, provided the original work is properly cited }\end{gathered}$

\title{
COMPARATIVE IN VITRO EVALUATION OF DIFFERENT BRANDS OF NIFEDIPINE 20mg RETARD TABLET PRODUCTS MARKETED IN ADDIS ABABA, ETHIOPIA
}

\author{
Girma Agune, Muluken Nigatu*, Tesfaye Gabriel, Abrham Temesgen, Yonas Brhane, Tesfa Marew \\ Department of Pharmaceutics and Social Pharmacy, School of Pharmacy, College of Health Sciences, Addis Ababa University, \\ Addis Ababa, Ethiopia
}

\begin{abstract}
Nifedipine has been formulated and marketed as extended-release-film coated tablet. A certain degree of success has been achieved in reducing the incidence of adverse effects by the use of slow-release formulations such as nifedipine retard. The aim of the present study was to evaluate the physicochemical quality attributes and in vitro equivalence of six brands of nifedipine retard tablets available in different retail outlets in Addis Ababa, Ethiopia. After constructing the calibration curve, the in vitro drug release studies were carried out using USP type I dissolution apparatus at $100 \mathrm{rpm}$. The dissolution was done in a medium of $0.1 \mathrm{~N} \mathrm{HCl}$ containing $0.5 \%$ sodium lauryl sulfate for $12 \mathrm{hrs}$. All the tablets met the requirement for tablet weight uniformity. The mean crushing strengths of sample tablets ranged from 49.2 to $111.2 \mathrm{~N}$. All the brands studied released more than $80 \%$ within 12 hours which is within the tolerance limit. However, the release profile revealed that five of the brands showed over $15 \%$ drug release at $1^{\text {st }}$ hour except product $\mathrm{F}$ which released only $14.32 \%$. In conclusion, all the brands of tablets had uniform thickness and good hardness. Despite all the brands had sustained the release for over 12 hours recommended for such formulations, five of them showed higher release in the first hour which may affect their in vivo performance.
\end{abstract}

Keywords: nifedipine, retard tablets, physicochemical properties, crushing strengths, in vitro drug release

Article Info: Received 19 Jan, 2018; Review Completed 19 March 2018; Accepted 20 March 2018; Available online 15 May 2018

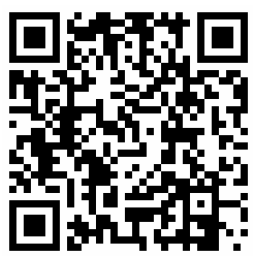

Cite this article as:

Agune G, Nigatu M, Gabriel T, Temesgen A, Brhane Y, Marew T, Comparative in vitro evaluation of different brands of nifedipine 20mg retard tablet products marketed in Addis Ababa, Ethiopia, Journal of Drug Delivery and Therapeutics. 2018; 8(3):1-5 DOI: http://dx.doi.org/10.22270/jddt.v8i3.1685

*Address for Correspondence: Muluken Nigatu, Department of Pharmaceutics and Social Pharmacy, School of Pharmacy, College of Health Sciences, Addis Ababa University, Addis Ababa, Ethiopia.

\section{INTRODUCTION}

Hypertension is a major public health problem worldwide with its attendant high rate of morbidity and mortality. Hypertension is a progressive disease that affects more than 1 billion people worldwide ${ }^{1,2}$. Reports showed that an estimated 639 million individuals had hypertension in developing countries in 2000 and this number is expected to rise to 1.15 billion by $2025^{3}$.

The primary goal of antihypertensive therapy is to control blood pressure and reduce the long-term risk of cardiovascular morbidity and mortality. Different classes of medication are available for the management of hypertension ${ }^{4,5,6}$. Nifedipine [Dimethyl-2,6-methyl-4( 2-nitrophenyl)-1, 4-dihydropyridine-3, 5- dicarboxylate] (Fig 1) is a calcium channel blocking agent which is commonly employed in the management of systemic hypertension and angina pectoris ${ }^{7}$.<smiles>COC(=O)C1=C(C)NC(C)=C(C(=O)OC)C1c1ccccc1[N+](=O)[O-]</smiles>

Figure 1: Chemical structure of nifedipine ${ }^{7}$

It has been demonstrated that the use of immediaterelease nifedipine oral formulations have been 
associated with rapid increase in nifedipine plasma concentration which results in increased heart rate and drug specific side effects such as flushing, dizziness, palpitation and reflex tachycardia. Accordingly, it is generally accepted that modified release formulations of nifedipine are the first therapeutic choice ${ }^{1,4,6.8,9}$.

Sustained release nifedipine is prepared as an extendedrelease-film coated tablet. A certain degree of success has been achieved in reducing the incidence of such adverse effects by the use of slow-release formulations such as nifedipine retard ${ }^{10,11,12}$.

Expiration of drug patents lead to several companies producing generic forms of drugs ${ }^{13}$. However, quality and performance of the generic versions of such drugs used in the management of chronic complications have been a source of debate among professionals and patients, particularly in the light of increasing circulation of counterfeited products and absence of strong regulatory systems in developing countries. The marketing of multisource drug products registered by national drug agencies in developing countries, with the view of improving health care delivery through competitive pricing, has an attendant problem of ascertaining their quality and interchangeability. As a result, health-care professionals sometimes pose questions whether these generics are equivalent to their original counterparts and whether patients are put at $\operatorname{risk}^{14,15}$.

The formulation of a tablet drug product can have a significant effect on its physicochemical quality parameters such as hardness, weight variation, disintegration time, dissolution profile which may in turn affect the in vivo performance. Hence, the present study was carried out to evaluate the physicochemical quality and in vitro equivalence of six brands of nifedipine retard tablets marketed by different retail outlets in Addis Ababa, Ethiopia.

\section{MATERIALS AND METHODS}

\section{Materials}

Six different brands of $20 \mathrm{mg}$ nifedipine retard tablets (Table 1) were purchased from retail outlets in Addis Ababa. All were film coated tablets except product C. Nifedipine reference standard was kindly donated by the Ethiopian Food, Medicine and Healthcare Administration and Control Authority (EFMHACA). Hydrochloric acid (BDH limited, Poole, England), HPLC grade methanol (Park Scientific Limited, UK), sodium lauryl sulfate, distilled water were used for the study. All chemicals used were analytical grade.

Table 1: Detailed description of products of nifedipine $20 \mathrm{mg}$ retard tablets included in the study

\begin{tabular}{|c|c|c|c|c|}
\hline Brand Code & Manufacturer & Country of origin & Batch no & Expiry date \\
\hline A & Cipla & India & GD61923 & $05 / 2019$ \\
\hline B & Fabricadop & Germany & 19021 & $08 / 2019$ \\
\hline C & Cadila & India & G603016 & $06 / 2019$ \\
\hline D & Remedica & Cyprus & 69778 & $09 / 2019$ \\
\hline E & E.I.P.I.Co & Egypt & 1509228 & $10 / 2018$ \\
\hline F & Cadila & India & D50025350 & $09 / 2017$ \\
\hline
\end{tabular}

\section{Methods}

\section{Measurement of thickness}

Ten tablets from each brand were taken and thickness was measured using sliding caliper scale (Nippon Sokutei, Japan). Results were expressed as a mean and standard deviation.

\section{Crushing strength}

Ten tablets were randomly selected from each brand product and the crushing strengths of the tablets were determined using hardness tester (Schleuniger, 2E/205, Switzerland). Each tablet was placed between two anvils and force was applied to the anvils, and the crushing strength that just caused the tablet to break was recorded. Results were expressed as a mean and standard deviation.

\section{Weight variation}

The weight variation test was evaluated by taking twenty tablets from each of the six brands, weighed individually with an analytical balance. The average weights for each brand as well as the percentage deviation from the mean value were calculated. Weight variation results were demonstrated as per USP (2013).

\section{Disintegration time}

Disintegration time test was carried out according to USP/NF (2013) specification. Six tablets were placed in a disintegration tester (CALEVA, G.B. Caleva Ltd., $\mathrm{UK}$ ) filled with distilled water at $37 \pm 0.5^{\circ} \mathrm{C}$. The tablets were considered completely disintegrated when all the particles are passed through the wire mesh and time was recorded.

\section{Calibration curve for Nifedipine RS}

Various concentrations of Nifedipine RS (17.5, 20, 25, $30,35,40,45$ and $50 \mu \mathrm{g} / \mathrm{ml}$ ) were prepared in a medium of $0.1 \mathrm{~N} \mathrm{HCl}$ containing $0.5 \%$ sodium lauryl sulfate and methanol. Absorbances were measured at $\lambda_{\max }$ of 329 $\mathrm{nm}$ using a UV-Visible spectrophotometer (SOLAR Spectrofluorimeter, CM2203, Belarus). The values of absorbance were plotted against the corresponding concentrations.

\section{In vitro drug release studies}

The in vitro drug release studies were carried out using USP type I dissolution apparatus (ERWEKA, DT600, Germany) at $100 \mathrm{rpm}$. The dissolution was done in a medium of $900 \mathrm{ml} 0.1 \mathrm{~N} \mathrm{HCl}$ containing $0.5 \%$ sodium lauryl sulphate for $12 \mathrm{hrs}$. The temperature was 
maintained at $37 \pm 0.5{ }^{\circ} \mathrm{C}$. Aliquot samples of $10 \mathrm{ml}$ were withdrawn at pre scheduled intervals $(1,3,4,6$, and $12 \mathrm{~h}$ ) and replaced with an equal volume of fresh dissolution medium which was kept at $37 \pm 0.5{ }^{\circ} \mathrm{C}$ to maintain sink condition. Each filtered sample was analyzed for drug content at $\lambda_{\max }$ of $329 \mathrm{~nm}$ using a UV/Visible Spectrophotometer.

\section{Statistical analysis}

Origin 7 Software (OriginLab Corporation, MA, and USA) was used to statistically analyze the results. All the data measured and reported are averages of a minimum of triplicate measurements and the values are expressed as mean \pm standard deviation.

\section{RESULTS AND DISCUSSION}

Tables 2 and 3 show some of the physicochemical characteristics of the nifedipine retard tablets studied. All tablets met the requirement (USP, 2013) for tablet weight uniformity and no tablet deviated from the average weight by more than $10 \%$ (samples A, C, D, E and F) and $7.5 \%$ (sample B). This compliance is important since the uniformity of dosage unit can be demonstrated by either weight variation or content uniformity study (USP/NF, 2013).

Table 2: Tablet weights of the nifedipine $20 \mathrm{mg}$ retard samples used in the study

\begin{tabular}{|ccccccc|}
\hline $\begin{array}{c}\text { Tablet } \\
\text { No }\end{array}$ & \multicolumn{5}{c|}{ Weight $(\mathbf{m g})$} \\
\cline { 2 - 7 } $\mathbf{1}$ & $\mathbf{A}$ & $\mathbf{B}$ & $\mathbf{C}$ & $\mathbf{D}$ & $\mathbf{E}$ & $\mathbf{F}$ \\
$\mathbf{2}$ & 83.0 & 181.7 & 94.4 & 87.1 & 93.0 & 112.8 \\
$\mathbf{3}$ & 83.1 & 176.4 & 95.0 & 86.0 & 92.3 & 110.4 \\
$\mathbf{4}$ & 81.6 & 173.8 & 95.4 & 87.5 & 94.2 & 111.8 \\
$\mathbf{5}$ & 83.6 & 170.0 & 94.6 & 88.3 & 93.5 & 110.6 \\
$\mathbf{6}$ & 84.7 & 175.8 & 96.0 & 87.8 & 93.5 & 110.3 \\
$\mathbf{7}$ & 84.4 & 176.0 & 95.8 & 87.7 & 95.3 & 111.9 \\
$\mathbf{8}$ & 82.2 & 175.3 & 94.6 & 87.2 & 96.2 & 111.4 \\
$\mathbf{9}$ & 85.5 & 177.3 & 95.2 & 86.0 & 94.4 & 110.8 \\
$\mathbf{1 0}$ & 84.7 & 174.5 & 94.4 & 88.4 & 94.3 & 112.6 \\
$\mathbf{1 1}$ & 84.7 & 172.9 & 96.2 & 87.7 & 94.9 & 112.8 \\
$\mathbf{1 2}$ & 82.2 & 179.3 & 95.8 & 90.0 & 95.9 & 112.9 \\
$\mathbf{1 3}$ & 84.3 & 170.8 & 97.3 & 87.6 & 94.7 & 109.9 \\
$\mathbf{1 4}$ & 84.0 & 175.3 & 94.7 & 86.9 & 88.0 & 116.0 \\
$\mathbf{1 5}$ & 83.5 & 175.2 & 94.3 & 86.7 & 91.5 & 110.6 \\
$\mathbf{1 6}$ & 82.7 & 177.5 & 94.4 & 87.9 & 97.3 & 11.7 \\
$\mathbf{1 7}$ & 83.6 & 177.1 & 94.1 & 87.6 & 91.1 & 107.6 \\
$\mathbf{1 8}$ & 82.4 & 177.0 & 96.6 & 88.5 & 94.6 & 111.8 \\
$\mathbf{1 9}$ & 82.2 & 174.5 & 94.5 & 87.6 & 93.7 & 110.3 \\
$\mathbf{2 0}$ & 84.7 & 181.0 & 95.1 & 88.3 & 93.3 & 111.4 \\
\hline
\end{tabular}

The tablet thickness ranged from 2.69 (product $\mathrm{D}$ and $\mathrm{E}$ ) to $3.43 \mathrm{~mm}$ (product $\mathrm{F}$ ). The mean crushing strengths of sample tablets ranged from 49.2 to $111.2 \mathrm{~N}$. Sufficient tablet hardness is essential to ensure resistance to damage by handling, packaging and transportation. Tablet hardness of $4 \mathrm{~kg}$ is considered to be the minimum for a satisfactory tablet ${ }^{12}$; hence all tablets conformed to the necessary requirements. Maximum and minimum crushing strengths were observed from product $\mathrm{D}$ and $\mathrm{A}$, respectively. Such differences in crushing strength may be resulted from different formulation and manufacturing technology.

Table 3: Some physicochemical characteristics of the nifedipine $20 \mathrm{mg}$ retard samples studied

\begin{tabular}{ccccc}
\hline Brand & $\begin{array}{c}\text { Tablet weight } \\
(\mathrm{mg})\end{array}$ & $\begin{array}{c}\text { Thickness } \\
(\mathrm{mm})\end{array}$ & $\begin{array}{c}\text { Crushing } \\
\text { strength }(\mathrm{N})\end{array}$ & $\begin{array}{c}\text { Disintegration } \\
\text { time }\end{array}$ \\
\hline $\mathrm{A}$ & $83.51 \pm 1.09$ & $2.98 \pm 0.02$ & $49.2 \pm 1.75$ & $5 \mathrm{~min} 10 \mathrm{sec}$ \\
$\mathrm{B}$ & $175.78 \pm 2.91$ & $3.26 \pm 0.06$ & $84.5 \pm 2.72$ & $45 \mathrm{sec}$ \\
$\mathrm{C}$ & $95.23 \pm 0.89$ & $2.88 \pm 0.05$ & $72.7 \pm 4.16$ & $56 \mathrm{sec}$ \\
$\mathrm{D}$ & $87.63 \pm 0.89$ & $2.69 \pm 0.02$ & $111.2 \pm 4.24$ & $1 \mathrm{~min} 50 \mathrm{sec}$ \\
$\mathrm{E}$ & $93.80 \pm 2.02$ & $2.69 \pm 0.05$ & $107.7 \pm 4.62$ & $3 \mathrm{hr} 54 \mathrm{~min}$ \\
F & $111.54 \pm 1.69$ & $3.43 \pm 0.04$ & $92.4 \pm 3.20$ & $>5 \mathrm{hr}$ \\
\hline
\end{tabular}

The disintegration time of the sample tablets showed great variation. Product $\mathrm{B}$ showed rapid disintegration time with only 45 seconds while with product $F$ which remained intact even after $5 \mathrm{hrs}$. Such lowest disintegration time of product $\mathrm{F}$ may suggest slower dissolution rate. 


\section{Construction of Calibration Curve}

The absorbance reading of nifedipine reference standard obtained was plotted against concentration (Figure 2). The linear regression equations obtained was $\mathrm{Y}=$ $0.01331 \mathrm{X}-0.01291\left(\mathrm{R}^{2}=0.9992\right)$ in $0.1 \mathrm{~N} \mathrm{HCl}$ containing $0.5 \%$ sodium lauryl sulfate and methanol where $\mathrm{Y}$ is absorbance and $\mathrm{X}$ is concentration in $\mu \mathrm{g} / \mathrm{ml}$.

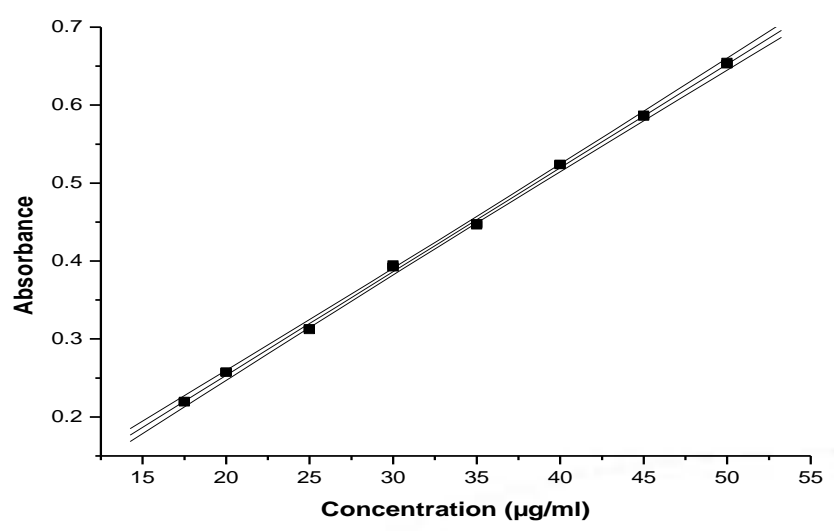

Figure 2: Standard calibration curve of nifedipine at $\lambda_{\max }$ of $329 \mathrm{~nm}$ in $0.1 \mathrm{~N} \mathrm{HCl}$ containing $0.5 \%$ sodium lauryl sulfate and methanol with upper and lower $95 \%$ confidence limits.

\section{In vitro drug release}

Bioequivalence studies are important to predict therapeutic equivalence between pharmaceutical equivalent test and reference products. In vitro dissolution studies have been recognized as important predictors of bioavailability for products on which formulation variables and processing parameters could have significant influence ${ }^{16,17}$.

Of the tests that can be performed on tablets, the dissolution test is considered to be sensitive, reliable and rational for predicting in-vivo drug availability behavior $^{18}$.

The drug release characteristics of dosage forms are usually tested by means of pharmacopoeial test methods under highly standardized conditions. These very well established methods are widely used as a tool for quality control and for the optimization of dosage forms ${ }^{9}$.

The result of drug release profile from the six brands of nifedipine retard tablets is illustrated in Figure 3. According to USP (2013), the acceptance limit for the amount of nifedipine released is given in Table 4. All the brands of nifedipine retard tablets studied released more than $80 \%$ within 12 hours which is within the tolerance limit. The release profile also revealed that five of brands showed more than $15 \%$ drug release at $1^{\text {st }}$ hour while product $\mathrm{F}$ released $14.32 \%$ within the $1^{\text {st }}$ hour. These results suggested that five of the studied brands (except F) exhibited higher initial drug release which may lead to dose dumping and compromise their therapeutic performance. Regarding the cumulative drug release within 4 hours, product F complied with USP dissolution tolerance limits $(39 \%)$ but all others showed more release $(>50 \%)$ than the stated amount within this period. Among all brands, product $\mathrm{F}$ had the least percentage release in the first 4 hours indicating its better retardant capacity than others.

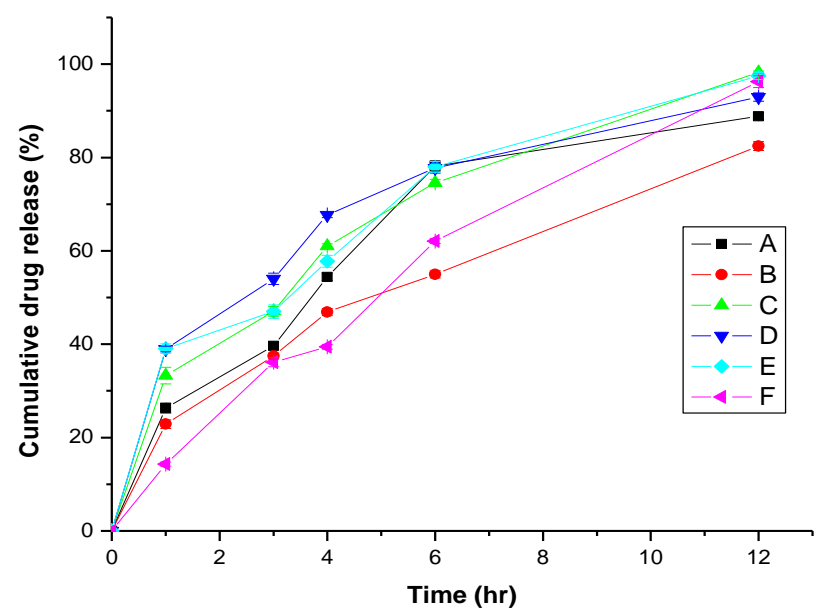

Figure 3: In vitro release profiles of nifedipine $20 \mathrm{mg}$ retard tablets

Table 4: The cumulative percentage released of the labeled amount of nifedipine retard tablets at specified time (USP 2013).

\begin{tabular}{cc}
\hline Time (hr) & Amount released (\%) \\
\hline 1 & Not more than $15 \%$ \\
4 & $20 \%-40 \%$ \\
12 & Not less than $80 \%$ \\
\hline
\end{tabular}

\section{CONCLUSION}

In the present study, 6 different brands of nifedipine 20 mg retard release tablets marketed in Addis Ababa were evaluated for different physicochemical properties. All the brands were found to have uniform thickness and weight and acceptable hardness. The results obtained were satisfactory and within the specified limits.

The first four brands were disintegrated within 15 minutes while product $\mathrm{E}$ and product $\mathrm{F}$ failed to disintegrate before 3 and 5 hours, respectively.

Based on the in-vitro dissolution studies, it was found that all brand products released more than $80 \%$ of the labeled amount within 12 hours in compliance with the USP tolerance limit. However, all brands except F released over $15 \%$ out of the acceptable monograph limit which may affect their in vivo performance. Similar pattern was observed up to $4 \mathrm{hrs}$ where five of the brands released over $50 \%$ above the USP recommended tolerance limit and only product $\mathrm{F}$ could meet the requirement.

Therefore, the results of the present study revealed that all the studied brands meet monogram specification for most of the physicochemical quality parameters but most of them (except F) failed to meet the $1^{\text {st }}$ and $4^{\text {th }} \mathrm{hr}$ USP in vitro dissolution tolerance limits which may affect the in vivo performance of these drugs.

\section{ACKNOWLEDGMENTS}

The authors are grateful to Addis Ababa University for providing us the opportunity to conduct the study and EFMHACA for kindly donating nifedipine reference standard. 


\section{CONFLICTS OF INTEREST}

Authors have declared that no conflicts of interest exist.

\section{ABBREVIATIONS}

EFMHACA: Ethiopian Food, Medicine and Health Care Administration and Control Authority

\section{REFERENCES}

1. Asare CO, Kipo SL, Kwakye KO, Gyasi MEB. Comparative in vitro dissolution of commercially available sustained release nifedipine tablet brands in the Kumasi Metropolis, Ghana. Journal of Applied Pharmaceutical Science; 2015; 5:54-60

2. Bloch MJ. Worldwide prevalence of hypertension exceeds 1.3 billion. Journal of the American Society of Hypertension; 2016; 10:753-754

3. Nshisso LD, Reese A, Gelaye B, Lemma S, Berhane Y, Williams MA. Prevalence of Hypertension and Diabetes among Ethiopian Adults. Diabetes Metab Syndr.; 2012; 6:36-41

4. Meredith PA, Elliott HL. A review of the gastrointestinal therapeutic system (GITS) formulation and its effectiveness in the delivery of antihypertensive drug treatment (focus on nifedipine GITS). Integrated Blood Pressure Control; 2013; 6:79-87

5. Shimamoto K, Kimoto M, Matsuda Y, Asano K, Kajikawa M. Long-term safety and efficacy of high-dose controlledrelease nifedipine (80mg per day) in Japanese patients with essential hypertension. Hypertension Research: 2015; 1-6

6. Snider ME, Nuzum DS, Veverka A. Long-acting nifedipine in the management of the hypertensive patient. Vascular Health and Risk Management; 2008; 4:1249-1257

7. Gajendran J, Kramer J, Shah VP, Langguth P, Polli J, Mehta M, Groot DW, Cristofoletti R, Abrahamsson B, Dressman JB. Biowaiver Monographs for Immediate-Release Solid Oral Dosage Forms: Nifedipine. Journal of Pharmaceutical Sciences; 2015; 104:3289 - 98

8. Akhter DT, Uddin R, Huda NH, Sutradhar KB. Design and Formulation of Twice Daily Nifedipine Sustained Release Tablet Using Methocel K15M CR and Methocel K100LV CR. Int J Pharm Pharm Sci; 2012; 4:121-124

9. Garbacz G, Golke B, Wedemeyer RS, Axell M, Soderlind E, Abrahamsson B, Weitschies W. Comparison of dissolution profiles obtained from nifedipine extended release once a day products using different dissolution test apparatuses.
USP: United States Pharmacopeia

USP/NF: United States Pharmacopoeia/National Formulary

UV: Ultraviolet

European Journal of Pharmaceutical Sciences; 2009; $38: 147-155$

10. Ghosh S, Ghosh NS, Debnath S, kumar GG, Chakraborty R, Sen S. Formulation and evaluation of sustained release dosage form of nifedipine hydrochloride using multi-unit chitosan treated alginate. IJPBR; 2010; 1:124-131

11. Minami J, Numabe A, Andoh $\mathrm{N}$, Kobayashi N, Horinaka S, Ishimitsu T, Matsuoka H. Comparison of once-daily nifedipine controlled-release with twice-daily nifedipine retard in the treatment of essential hypertension. $\mathrm{Br} J$ Clin Pharmacol; 2004; 57:632-639

12. Okoye EI, Iwuagwu MA. Physicochemical equivalence of some brands of Nifedipine retard tablets available in Nigeria. Afr. J. Biotechnol.; 2010; 9:1274-1279

13. Muaz J, Gazali LK, Sadiq GU,Tom GM. Comparative in vitro evaluation of the pharmaceutical and chemical equivalence of multi-source generic ciprofloxacin hydrochloride tablets around Maiduguri metropolitan area. Nig. Journ. Pharm. Sci.; 2009; 8:102 - 106

14. Akarawut W, Suvakontha T, Poompanich A. Pharmaceutical Quality of Nifedipine Soft Capsules Commercially Available in Thailand. Journal of Health Science; 2002; 11:1-8

15. Awofisayo SO, Awofisayo OA, Eyen N, Udoh IE Comparative Assessment of the Quality Control Measurements of Multisource Ofloxacin Tablets Marketed in Nigeria. Dissolution Technologies; 2010; 17:20-25

16. CDSCO. Guidelines for Bioavailability \& Bioequivalence Studies, Central Drugs Standard Control Organization (CDSCO), Directorate General of Health Services, Ministry of Health \& Family Welfare, Government of India, New Delhi. 2005

17. Ilic M, Kovacevic I, Parojcic J. Deciphering nifedipine in vivo delivery from modified release dosage forms: Identification of food effect. Acta Pharm.; 2015; 65:427-441

18. Poonguzhali S, Anusha K, Mounica T, Niharika PML, Kumar MS, Nadendla R. Comparative in vitro Evaluation of Commercial Atenolol Tablets. RJPBCS 2014; 5(6):30-35 